Europhys. Lett., 72 (6), pp. 990-996 (2005)

DOI: $10.1209 / \mathrm{epl} / \mathrm{i} 2005-10339-0$

\title{
Onset of rigidity for stretched string networks
}

\author{
G. W. Delaney, D. Weaire and S. Hutzler \\ School of Physics, Trinity College Dublin - Dublin 2, Ireland
}

received 10 August 2005; accepted in final form 21 October 2005

published online 23 November 2005

PACS. 64.60.Ak - Renormalization-group, fractal, and percolation studies of phase transitions.

PACS. 45.70.Cc - Static sandpiles; granular compaction.

PACS. 07.05.Tp - Computer modeling and simulation.

\begin{abstract}
We define and analyse an elementary model for a network of strings with random natural lengths. As this system is expanded, a threshold is reached where some of the strings form a taut string network. Further expansion increases the fraction of taut strings, until eventually all strings are taut and the classical problem of a spring network is recovered. Here we provide an analysis of the properties of the system at and beyond the threshold expansion, relating results to the expectations of constraint theory.
\end{abstract}

Introduction. - We will define and analyse an elementary model which has a close affinity to existing models that represent the compression of randomly packed soft spheres, often invoked as a description of liquid foams $[1,2]$ and randomly cut elastic strings. It therefore belongs to the subject of "rigidity percolation" [3]. It appears to be distinct from either of its antecedents, and not trivially related to them. It was originally conceived as a variation on the theme of soft disk and sphere packings, as formulated by Liu and others [4-6]. That work is mainly founded on Hooke's law interactions which act under compression only (similar to those found for example in the interaction between the spheres in a Newton's cradle [7]). It has proved to be extremely rich in subtle phenomena, such as non-integer indices that relate contact number variations with compression. It occurred to us to simply turn this problem "inside-out", by defining Hooke's law interactions under extension only, and hence a model of elastic strings that are loose under compression. The strings initially connect nearest-neighbour vertices of a regular lattice and are given natural lengths $l_{i}$ that are random variables. Here we will use a uniform distribution for $l_{i}$. We may ask: when and how does the resulting network become taut as its boundaries are expanded? This is analogous to the "jamming" problem of compressed hard spheres, but simpler in some respects, as we shall see.

We have simulated the above system for large numbers of vertices in two dimensions, using periodic boundary conditions. Results for different lattices are quite distinct, depending on their coordination number. Here we will consider the cases of the hexagonal, square and triangular networks, with each vertex having three, four and six neighbours, respectively. 


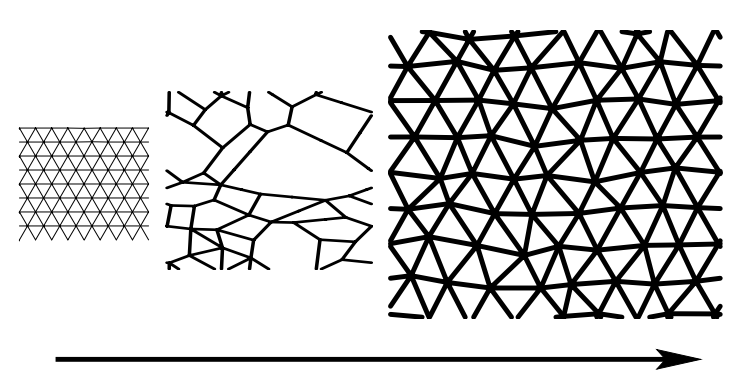

(a)

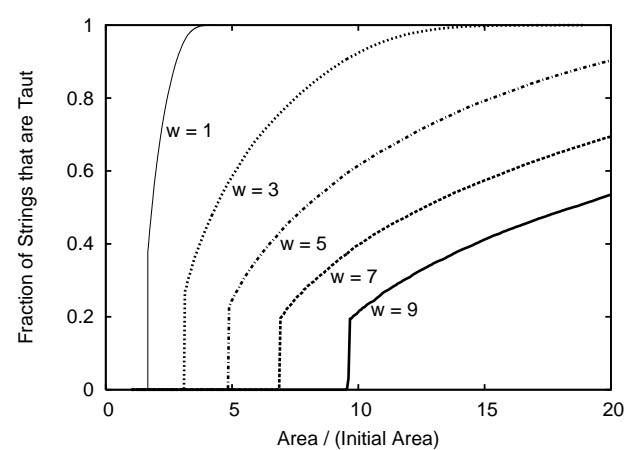

(b)

Fig. 1 - (a) Expansion of a triangular lattice with 192 strings and range $w=2$ for the natural length of the strings. In the initial configuration of strings (left) all strings are slack and represented by thin lines between vertices. As the system is expanded, a fraction of the strings become taut. Only these taut strings are shown as thick black lines, close to the threshold expansion (middle) and for the expansion where the last spring becomes taut (right). (b) Increase in the number of taut strings as the periodic box is expanded, for a triangular lattice with 49152 strings. Data is shown for five different ranges of random natural lengths of the strings $w=1, w=3, w=5, w=7$ and $w=9$.

Simulation technique. - We have used the Surface Evolver software [8] to define our string network system with periodic boundary conditions and to minimise the energy of the structure with a highly optimised conjugate gradient routine. We have modified the source code to redefine the Hookean spring energy as one-sided:

$$
E_{i}= \begin{cases}0 & \text { if } L_{i} \leq l_{i} \\ k\left(L_{i}-l_{i}\right)^{2} & \text { if } L_{i}>l_{i}\end{cases}
$$

where $L_{i}$ is the separation of the two vertices at the ends of the string $i$ and $l_{i}$ is the natural length of each of the $N$ strings. For $L_{i}<l_{i}$ a string is slack.

We define a string network, consisting of strings and vertices (a string is formed by joining two vertices) in a two-dimensional box with periodic boundary conditions. The lattice is initially defined with all nearest-neighbour separations equal to unity. The natural lengths $l_{i}$ of the corresponding $N$ elastic strings are uniformly distributed in the range

$$
1 \leq l_{i} \leq l_{\max } .
$$

The width of the distribution of random numbers is thus given by $w=l_{\max }-1$. The system is uniformly expanded by a small amount using an affine transformation. The structure is then relaxed to minimise the total energy. This procedure of small expansion followed by relaxation is continued until all strings have become taut (fig. 1). A fraction of the strings first become taut at some previous point, which we may call the threshold of rigidity.

In considering the basic properties of the model, we recognise some of its simplifying features. In contrast to the soft-sphere problem, this stretched string model has the property of convexity, as does the line minimisation problem [9]. Convexity is defined (in this case) as follows. At every point in the space of vertex coordinates, the second derivative of energy 


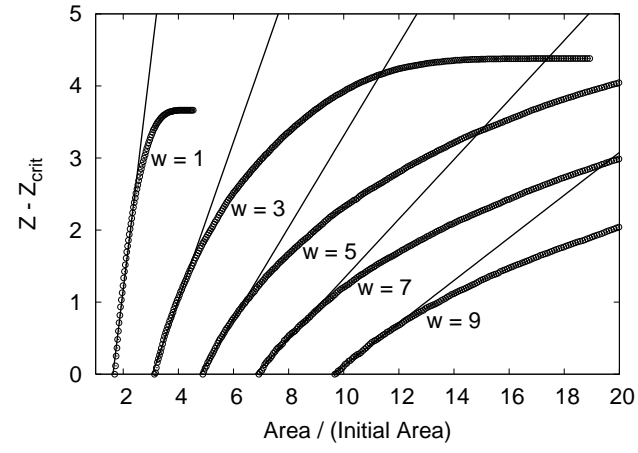

(a)

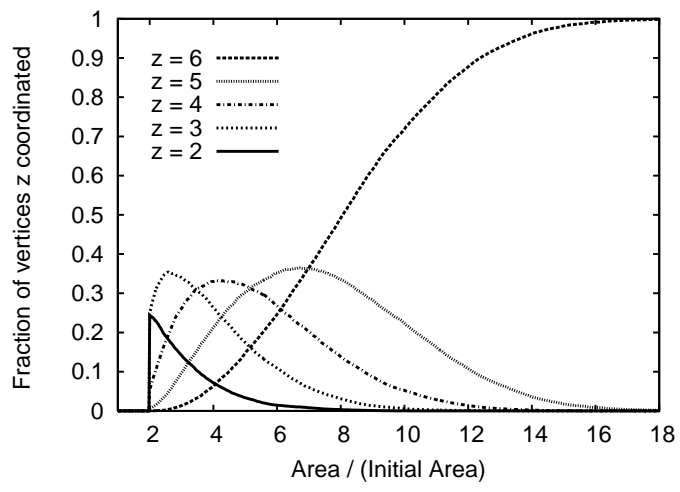

(b)

Fig. 2 - Triangular lattice (49152 strings) above the threshold of rigidity. (a) As the network is expanded, the average coordination number $Z$ increases linearly close to its threshold value, $Z_{\text {crit }}$ (the data shown is for five different ranges $w$ of random natural lengths of strings, the lines are linear least squares fits to the data close to the threshold). (b) Variation in the fraction of vertices with coordination numbers $z=2$ to $z=6$ as the network is expanded (here $w=4$ ).

with respect to displacement is non-negative for all directions. This follows easily from the same statement applied to the energy term associated with each string.

Convexity implies that there cannot be more than one disjoint minimum of energy at and above the threshold expansion. (A sketch of a simple curve with two minima makes this obvious.) Unlike many of its disordered cousins, the model has a unique global minimum of energy. Thus, any stable state reached in our minimisation of the system is the unique solution for the string network. This reinforces the point made earlier, to the effect that turning the soft-sphere model inside-out entails quite different properties, since the soft-sphere model has a multiplicity of alternative minima.

In this letter we present results for very large systems with $N=32178$ for the square case and $N=49152$ for the triangular and hexagonal case. We find the same system behaviour when we consider systems with smaller numbers of strings. Also for the large systems considered here, there do not appear to be any appreciable finite-size effects.

Simulation results for the triangular lattice. - For the ordered case $(w=0)$, it is obvious that expanding the boundaries simply results in an affine expansion of the lattice, all strings becoming equally taut immediately. However, even quite small values of $w$ produce radically different initial behaviour upon expansion. In such a case, at a certain critical expansion the system becomes taut, with an average coordination number $Z_{\text {crit }}$ (average number of taut strings connected to a vertex), which we shall examine.

As the boundaries are further expanded beyond this point, the average coordination number $Z$ increases. It is obvious that for a finite-width distribution of $l_{i}, Z$ should tend to six (all strings taut) as the expansion tends to infinity; indeed it is clear that this should occur at some finite expansion. Its initial variation beyond the critical expansion is less obvious; at what rate do the loose strings become taut? We find this variation to be linear for the triangular case (fig. 2(a)). This is a very different behaviour from the strong nonlinear effects for disk and sphere packings under compression, as represented by compressed springs. In 


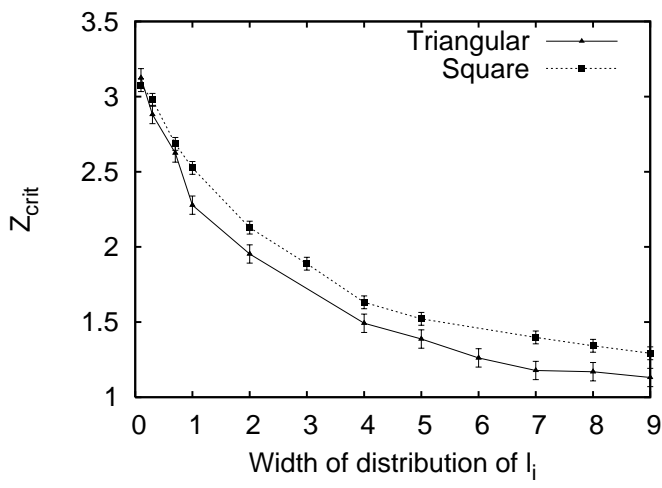

(a)

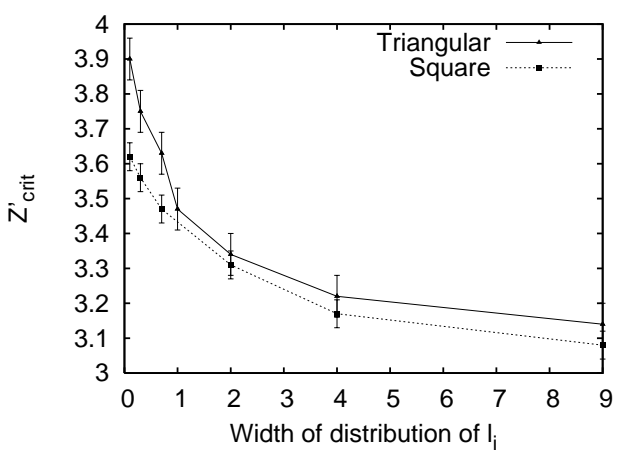

(b)

Fig. 3 - (a) Variation of the average coordination number $Z_{\text {crit }}$ at the threshold of rigidity as a function of the width $w$ of the distribution of random natural lengths $l_{i}$. (b) Disregarding vertices with coordination $z \leq 2$ leads to a value of $Z_{c r i t}^{\prime}$ which in the limit of $w \rightarrow 0$ is much closer to four, which is the prediction of constraint theory. (The data shown is for a square lattice with 32768 strings and a triangular lattice with 49152 strings.)

that case a power law variation with exponent 0.5 is found for the variation of the variation of excess contacts with packing fraction above the jamming threshold [6].

An individual vertex can have a coordination number $z$ of taut connections between $z=2$ and $z=6$. Figure $2(\mathrm{~b})$ shows the variation in the fraction of vertices with each coordination number as the system is expanded. At the threshold expansion the coordination number of some of the vertices jumps up from zero. Initially, there is a large number of vertices with coordination $z=2(24.3 \%$ for the case with $w=4$ shown in fig. 2(b)). This decreases steadily as the system is expanded and more strings become a part of the taut network. We see a similar decrease for $z=3$. Coordination numbers $z=4$ and $z=5$ show an initial increase and then decrease for larger expansions as the system approaches the fully taut state where all vertices have $z=6$.

Figure $3(\mathrm{a})$ shows the variation of the average coordination number $\left(Z_{\text {crit }}\right)$ at the threshold expansion with the range of the natural string lengths $w$. The steep initial descent of $Z_{\text {crit }}$ makes it difficult to extrapolate reliably for $w \rightarrow 0$. It is consistent with a finite value less than 6 . There also appears to be a monotonic decrease of $Z_{\text {crit }}$ as $w$ tends to infinity.

Interpretation. - The first key to understanding these results is surely constraint theory, a venerable mathematical technique with important modern applications in areas such as protein flexibility [10] and the random packing of particles [11]. It seeks to identify constraints and degrees of freedom, and equates these to make estimates of the statistics of critical points of the general kind that we have encountered here. In the triangular case, each vertex has the two degrees of freedom of translation and each taut string, maintained at its natural length $l_{i}$, will contribute one constraint which is $L=l_{i}$. The expectation is therefore that, in the absence of any symmetry (such as that which one obtains when $l_{\max }=1$ ), the degrees of freedom are exhausted when the average coordination $Z=4$, since each string joins two vertices. However, we see that the estimate from constraint theory appears not to be at all accurate even for small values of $w$ (the range of natural lengths). For $w=0.1$, the average 


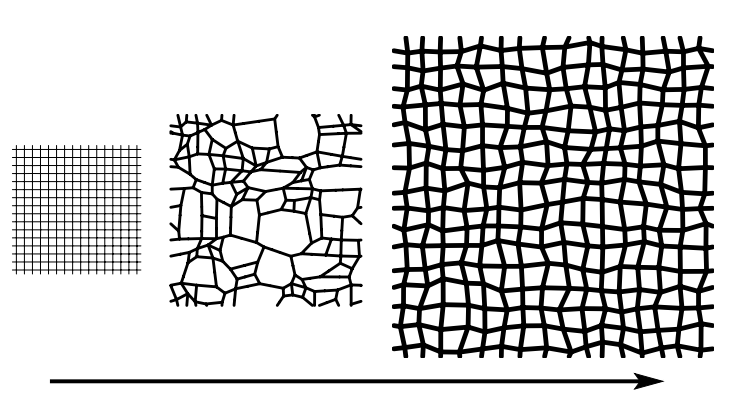

(a)

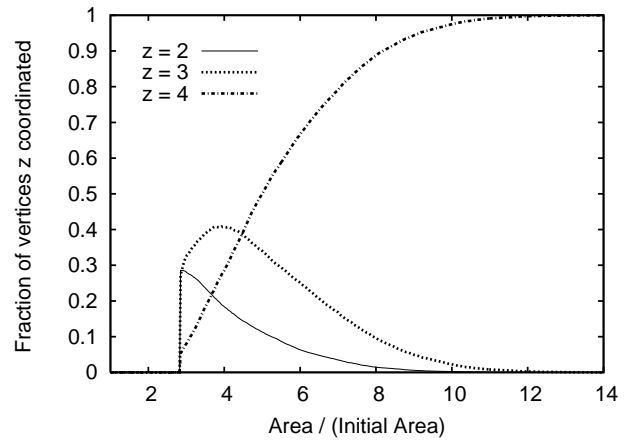

(b)

Fig. 4 - Expansion of a square lattice. (a) Simulation output for 512 strings and $w=2$, displayed as in fig. 1. (b) Variation in the fraction of vertices with coordination numbers $z=2$ to $z=6$ as the system is expanded (the data shown is for a square lattice composed of 32768 strings and $w=4$ ).

coordination at the threshold is $Z_{\text {crit }}=3.12 \pm 0.06$. For larger values of $w$ there is a strong decrease of $Z_{\text {crit }}$, which appears to level off for large values of $w$.

We see that even for $w \rightarrow 0$, the naive estimate from constraint theory $\left(Z_{\text {crit }}=4\right)$ is not found, and the discrepancy grows rapidly with $w$. The reason for this lies largely in the symmetry of the initial state. It can happen that a vertex is connected to only two taut strings, in which case it should be considered redundant in any constraint arguments, and the two strings considered as one. (This is obvious as taut strings that join vertices with coordination $z=2$ must lie collinear and in effect behave as a single string.) We can also disregard all vertices that are only connected to slack strings $(z=0)$, as these do not contribute to the taut network. With these refinements and thus a definition of an average coordination number $Z^{\prime}$ which does not take into account vertices with $z \leq 2$, the constraints argument does succeed, as fig. 3(b) shows. We find that $Z_{c r i t}^{\prime}=3.9 \pm 0.06$ for $w=0.1$.

This correction does not suffice to explain the additional decrease of $Z_{\text {crit }}$ at high $w$, and a further analysis would be required to see how this comes about.

Results for the square lattice. - A square lattice is set up with each vertex connected to its four nearest neighbours. An example of a simulation using a square lattice is shown in fig. 4(a). This is an interesting lattice configuration, as it has the same number of nearestneighbour connections as the number of constraints predicted to be required for the system to become taut. Nevertheless, the system behaves in a somewhat similar manner to the triangular case. As the system is expanded, a threshold expansion is reached where a fraction of the strings become taut. As the system is further expanded there is an initial linear increase in the fraction of strings that are taut.

For a square lattice, a constrained vertex can have a coordination number between $z=2$ and $z=4$. Figure 4(b) shows the variation in the fraction of vertices with each coordination number as the system is expanded. At the threshold expansion, the first strings become taut and so the coordination of some of the vertices jumps up from zero. We see again that a large fraction of vertices have coordination $z=2(28.5 \%$ for the case with $w=4$ shown in fig. 2(b)). This decreases steadily as the system is expanded and more strings become part 


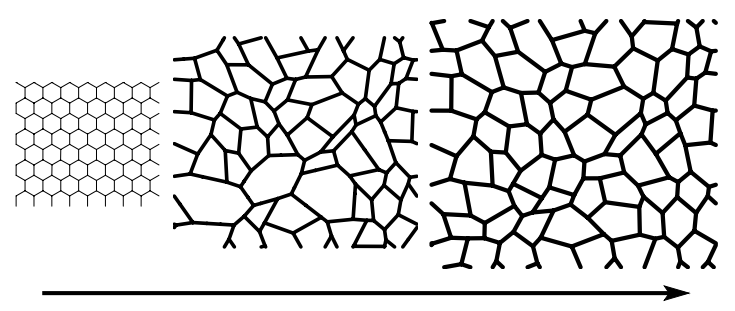

(a)

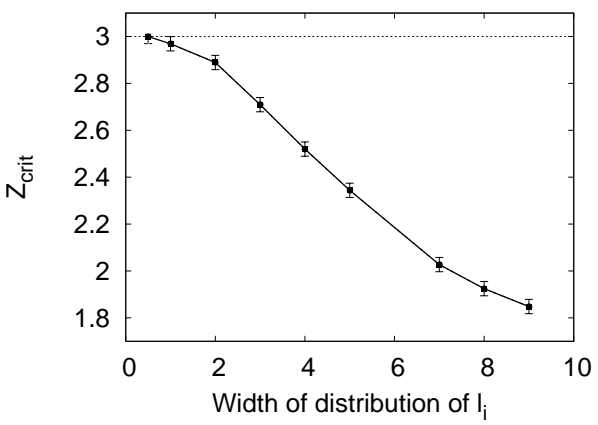

(b)

Fig. 5 - Expansion of a hexagonal lattice. (a) Simulation output for 192 strings and $w=2$, displayed as in fig. 1. (b) Variation of the average coordination number $Z_{\text {crit }}$ at the threshold of rigidity as a function of the width $w$ of the distribution of random natural lengths $l_{i}$ (the data shown is for a hexagonal lattice with 49152 strings). As the maximum coordination is 3 , disregarding all vertices with coordination $z \leq 2$ gives a constant value of $Z_{\text {crit }}^{\prime}=3$, indicated by the dashed line.

of the taut network. The fraction of vertices with $z=3$ shows an initial increase and then decrease for larger expansions, a similar behaviour as for $z=4$ and $z=5$ for the triangular lattice. Finally, as the system approaches the fully taut state all vertices tend to $z=4$.

The variation of coordination number $\left(Z_{\text {crit }}\right)$ at the threshold expansion with the distribution of natural lengths $w$ is shown in fig. 3(a). Here again we see a steep initial descent of $Z_{\text {crit }}$ which makes it difficult to extrapolate reliably the limit $w \rightarrow 0$. It is consistent with a finite value less than 4 . There also appears again to be a monotonic decrease of $Z_{\text {crit }}$ as $w$ tends to infinity, similar to what is seen in the triangular case.

It is clear from fig. 4(a) (and from the large number of vertices with coordination $z=$ 2 in fig. 4(b)) that even at the threshold expansion we again have substantial pockets of loose strings. When we calculate the coordination number of the taut network $\left(Z_{\text {crit }}^{\prime}\right)$ as for the triangular case, we again find that in the low- $w$ limit results are brought towards the predictions of constraint theory with $Z_{\text {crit }}^{\prime}=3.62 \pm 0.04$ for $w=0.1$, but not as closely as in the triangular case.

Results for the hexagonal lattice. - A hexagonal lattice is set up with each vertex connected to its three nearest neighbours. An example of a simulation using a hexagonal lattice is shown in fig. 5(a). This lattice configuration has one fewer nearest-neighbour connections than the predicted number of constraints required for the system to become taut. We would thus expect that as the system is expanded, a threshold will be reached where all strings would become taut simultaneously and that this behaviour would be independent of the width of the distribution of random lengths $w$.

We see in fig. 5(b) that the system behaves in the expected manner only in the low- $w$ limit. As $w$ is increased, the average coordination $Z_{\text {crit }}$ at the threshold decreases as for the triangular and square lattices. The coordination number of the taut network $\left(Z_{\text {crit }}^{\prime}\right)$ formed at the threshold is indicated by the dashed line in fig. $5(\mathrm{~b})$. This must always be $Z_{\text {crit }}^{\prime}=3$ as by definition disregarding vertices with $z \leq 2$ in a system where the maximum coordination number is 3 , must only leave the vertices with coordination $z=3$. 
Conclusion. - A network of elastic strings of random lengths under tension has been seen here to behave in a very different way to the case of elastic disk and sphere packings under compression. In particular there is a global minimum of energy above threshold. There is a strong dependence of the behaviour of the system, and in particular the critical average coordination number, on the polydispersity (represented by the distribution of natural lengths of the strings). The naive application of constraint theory requires various corrections to explain these results.

There are also instances of the failure of constraint theory for packings of objects in two and three dimensions. Packings may for example contain "rattlers", objects that can move freely in the interstices of a rigid system of larger scale. A naive application of constraint theory also runs into difficulty in the case of objects that have an asymmetric shape $[11,12]$.

In future extensions to this work we will deal with other lattices and other significant variables that characterise their behaviour. Of particular interest are the elastic properties of the network and a statistical analysis of the pockets of loose strings. Since the model corresponds closely to an experimental system that can be directly realised, the construction of an experimental system could also be considered.

$$
* * *
$$

Research was supported by Enterprise Ireland (BRGS SC/2002/011). GD holds a Trinity College Dublin Postgraduate Award. We also gratefully acknowledge computational support from the Trinity Centre for High Performance Computing and the Irish Centre for High-End Computing.

\section{REFERENCES}

[1] Durian D. J., Phys. Rev. Lett., 75 (1995) 4780.

[2] Weaire D. and Hutzler S., The Physics of Foams (Oxford) 1999.

[3] Jacobs D. J. and Thorpe M. F., Phys. Rev. E, 53 (1996) 3682.

[4] Liu A. J. and Nagel S. R., Nature, 396 (1998) 21.

[5] O'Hern C. S., Langer S. A., Liu A. J. and Nagel S. R., Phys. Rev. Lett., 88 (2002) 075507.

[6] O'Hern C. S., Silbert L. E., Liu A. J. and Nagel S. R., Phys. Rev. E, 68 (2003) 011306.

[7] Hutzler S., Delaney G., Weaire D. and Macleod F., Am. J. Phys., 72 (2004) 1508.

[8] Brakke K, Exp. Math., 1 (1992) 141.

[9] Durand M., Sadoc J. F. and Weaire D., Proc. R. Soc. London, Ser. A, 460 (2004) 1269.

[10] Thorpe M. F., Lei M., Rader A. J., Jacobs D. J. and Kuhn L. A., J. Mol. Graph. Model., 19 (2001) 60.

[11] Delaney G., Weaire D., Hutzler S. and Murphy S., Philos. Mag. Lett., 85 (2005) 89.

[12] Donev A., Cisse I., Sachs D., Variano E., Stillinger F. H., Connelly R., Torquato S. and Chaikin P. M., Science, 303 (2004) 990. 